\title{
Anspruch und Wirklichkeit beim Umbau der außeruniversitären Forschung nach der Wende
}

\author{
Das Beispiel des Forschungsschwerpunkts Moderner Orient
}

\section{Eine Tagung zur Wissenschaftsgeschichte in Berlin}

Die Berliner Wissenschaftsgeschichte ist reich an historischen Ereignissen und Prozessen. Immer wieder gibt es Gelegenheit, darüber nachzudenken und zu diskutieren. So stand im Dezember 2012 in einem Workshop die Geschichte einer Berliner Wissenschaftsinstitution zur Diskussion, die als eine relativ junge Einrichtung zu bezeichnen ist. Das Geisteswissenschaftliche Zentrum (GWZ) Moderner Orient hatte eingeladen, um unter dem Titel »Geteilt - vereint - global. Vom Forschen im geteilten Deutschland zum Forschen ohne Grenzen « die hauptstädtische Geschichte der Afrika-, Asien- und Orientforschung zu diskutieren, was letztlich bedeutete, sich mit der eigenen Geschichte zu befassen.

Neben Wissenschaftshistorikern sowie ehemaligen und gegenwärtigen Mitarbeitern des Zentrums waren auch einige Wissenschaftspolitiker zugegen, die vor mehr als 20 Jahren in unterschiedlicher Weise zur Entstehung dieser außeruniversitären Wissenschaftseinrichtung beigetragen hatten.

Das ZMO, wie die Institution abgekürzt gern genannt wird, entstand aus dem »Forschungsschwerpunkt Moderner Orient« (FSP) - einer Zwischenetappe der Abwicklung der Akademie der Wissenschaften (AdW) der DDR nach der Vereinigung der beiden deutschen Staaten. Der Personalbestand und die Ideen für verschiedene im FSP und später im ZMO zu bearbeitende Projekte stammten aus der Erbmasse des Instituts für Allgemeine Geschichte der AdW, in dem auch Afrika-, Asien- und Orientwissenschaftler arbeiteten und deren Forschungen eine bis ins 19. Jahrhundert zurückreichende Tradition aufweisen.

Im Rückblick ist es schon verwunderlich, dass einem Teil von ihnen - im Gegensatz zur Masse der an der AdW arbeitenden Historiker - die Chance eingeräumt worden war, ihre Forschungen weiter betreiben zu können. Dies war seinerzeit nicht unumstritten. Wohl auch deshalb hatte sich die Arbeitsgruppe »Wissenschaft und Wiedervereinigung « der Berlin-Brandenburgischen Akademie der Wissenschaften, die sich unter Leitung von Jürgen Kocka mit der Geschichte einzelner Wissenschaftsdisziplinen in der DDR beschäftigte, in der ersten Hälfte der 1990er Jahre mit den Afrika-, Asien- und Orientwissenschaften an der AdW befasst. ${ }^{1}$ Kurz zusammengefasst: Die Arbeitsgruppe hatte es sich unter anderem zur Aufgabe

1 Vgl. Krauth, Wolz 1998.

Leviathan, 41. Jg., 4/2013, S. 511 
gemacht, zu eruieren, welche wissenschaftliche Qualität ausgewählte Wissenschaftsdisziplinen, insbesondere geisteswissenschaftliche Disziplinen, aufwiesen oder inwieweit diese das politische System der DDR lediglich stützen oder legitimieren sollten. ${ }^{2}$

Zu diesem Zeitpunkt waren die Asien-, Afrika- und Lateinamerikawissenschaftler schon bis auf einen kleinen Rest von ungefähr zehn Wissenschaftlern »wegevaluiert « worden. Es ging also nur noch einmal um eine nachholende Bestandsaufnahme. Manchen westdeutschen Evaluierern war wohl im Zuge der Bewertung ostdeutscher Kollegen die Erkenntnis gekommen, dass die unerbittliche Zerschlagung der geisteswissenschaftlichen Projekte und Strukturen und die damit im Zusammenhang stehende Entlassung der Akademiker keine gute Lösung sei und die Gefahr bestünde, dass man später feststellte, dass zumindest in vielen Fällen zu rigoros geurteilt wurde. Diese Erkenntnis entstand jedoch erst in einem langen Prozess des Nachdenkens und lässt sich auch nur bei einigen Kollegen nachweisen. So stellte der ehemalige Vorsitzende des Wissenschaftsrats, Dieter Simon, in der Wochenzeitung Die Zeit 1995 fest, dass man im Prozess der deutschen Einheit den großen Fehler gemacht habe, das "marode westdeutsche Wissenschaftssystem " einfach in den Osten zu übertragen und »Ruinen aus dem Westen als kostspielige Modelle für den Aufschwung « hinzustellen. Auf diese Weise habe man die Wissenschaft der DDR »verschleudert und verschludert . $^{3}$

Nach mehr als zwei Jahrzehnten deutscher Einheit ist es daher vielleicht angebracht, an einem Beispiel zu überprüfen, was mit der Evaluation der DDR-Wissenschaftler zu Beginn der 1990er Jahre bezweckt worden war, was aufgrund der Evaluationsberichte vom Wissenschaftsrat vorgeschlagen wurde und ob und wie diese Empfehlungen in Bezug auf den Forschungsschwerpunkt Moderner Orient beziehungsweise dem späteren Geisteswissenschaftlichen Zentrum Moderner Orient letztendlich umgesetzt worden sind. Immerhin lässt sich an solchen Beispielen nachweisen, inwieweit Empfehlungen des höchsten wissenschaftlichen Beratungsorgans der Bundesrepublik - der Wissenschaftsrat - reale Umsetzung in der Vergangenheit fanden.

Mit dem Artikel 38 des deutschen Einigungsvertrags war unter anderem die Aufgabe der »Einpassung « der wissenschaftlichen Einrichtungen der DDR in die Forschungsstruktur der Bundesrepublik Deutschland erteilt worden. Der Wissenschaftsrat hatte Kriterien für eine solche Einpassung erstellt. Auch eine gesonderte Stellungnahme für die geisteswissenschaftlichen Institute der Akademie der Wissenschaften hatte der Wissenschaftsrat erarbeitet. Diese orientierte sich an dem Ziel, »die Entstehung einer in sich verträglichen Wissenschaftsstruktur in Deutschland zu fördern «. ${ }^{4}$ In einem »Parforcegang ", so eine spätere Beschreibung, ${ }^{5}$ hatte der Wissenschaftsrat die Evaluierung der DDR-Wissenschaftsinstitutionen in seinem

2 Vgl. Kocka, Mayntz 1998.

3 Simon 1995.

4 Wissenschaftsrat 1991, S. 5.

5 Schattenfroh 1998. 
»prominentesten Fall « 6 - so der damalige Vorsitzende des Wissenschaftsrats, Dieter Simon, in der Rückschau - übernommen. Mit welchem Ergebnis?

\section{Vom Forschungsschwerpunkt zum Geisteswissenschaftlichen Zentrum Moderner Orient}

Anhand der Ergebnisse der Evaluation der geisteswissenschaftlichen Institute der Akademie der Wissenschaften der DDR legte der Wissenschaftsrat Empfehlungen vor, die weitreichende Folgen insbesondere für geisteswissenschaftliche außeruniversitäre Forschungseinrichtungen im Osten des wiedervereinigten Deutschlands hatten. Wenn auch von vielen kritisiert - nicht zuletzt von denjenigen ostdeutschen Forschern, die durch die Empfehlungen der fast ausnahmslos westdeutsch besetzten Evaluationskommission ihre Stellen verloren -, so stellten die Empfehlungen doch etwas Neues, das vereinte Deutschland Bereicherndes, in der bisherigen außeruniversitären Wissenschaftslandschaft der Bundesrepublik ungewöhnlich Kreatives dar. Vor allem hatten sich die Mitglieder der Evaluierungskommissionen bemüht, die Ergebnisse der wissenschaftlichen Arbeiten und die guten Vorleistungen ostdeutscher Forscher, etwa in der außereuropäischen Sozialgeschichte oder der Kolonialhistoriographie, anzuerkennen.

Personell zwar stark reduziert, wurde aber in Anerkennung der akademischen Kreativität und mit der Bereitschaft, an etwas Neuem mitzuwirken, eine Schar meist jüngerer Wissenschaftler in die dafür geschaffene Förderungsgesellschaft Wissenschaftliche Neuvorhaben, einer Tochtergesellschaft der Max-Planck-Gesellschaft (MPG), übernommen. Diese Tochtergesellschaft bestand aus sieben aus dem Akademie-Nachlass entstandenen Forschungsschwerpunkten. Es war eine kühne und innovative Idee besonders deshalb, weil die Eliten der Bundesrepublik sich in der Vereinigung der beiden Länder als Sieger der Geschichte empfanden.

Wären die Empfehlungen des Wissenschaftsrats tatsächlich umgesetzt worden, so sähe die schon damals von einigen Geisteswissenschaftlern der alten Bundesrepublik kritisierte Wissenschaftslandschaft Deutschlands heute anders, vor allem vielfältiger aus. Doch dazu kam es nicht.

Die aus der Evaluation gezogenen Schlussfolgerungen, die in den Wissenschaftsrats-Empfehlungen zu Papier gebracht wurden, sollten verhindern, dass die Probleme und Defizite in den Geisteswissenschaften, die damals gerade von einigen führenden Geisteswissenschaftlern der alten Bundesrepublik durch eine Publikation öffentlich gemacht worden waren, ${ }^{7}$ reproduziert werden. Alte Fehler sollten bei der Neugestaltung der Wissenschaft in den neuen Bundesländern so weit wie möglich vermieden werden. Der Vorschlag zur Gründung von Forschungsschwerpunkten eröffnete eine solche Möglichkeit.

Zur Erarbeitung der entsprechenden Vorschläge hatte der Wissenschaftsrat eigens hierfür zusammengestellte Expertengruppen eingesetzt, welche die in der abge-

6 Simon 1997.

7 Frühwald et al. 1990.

Leviathan, 41. Jg., 4/2013 
schotteten DDR von ihren ostdeutschen Kollegen erbrachten wissenschaftlichen Leistungen auswerteten. Jedoch, "es waren Gremien ", so beschreibt der NahostExperte Martin Robbe den Vorgang, »in denen Wissenschaftler aus den alten Bundesländern das Sagen hatten, die das wissenschaftliche Potential der Ex-DDR evaluierten und entschieden, was mit ihm zu geschehen habe. Die Betroffenen wurden kaum in die Entscheidungsfindung einbezogen, so dass auch ihre [nach der Wende erarbeiteten - U.v.d.H.] konzeptionellen Vorstellungen weithin unbeachtet blieben ${ }^{8}{ }^{8}$

\section{3. (K)Ei(ge)ne Vorstellungen?}

Schon im Prozess der deutschen Vereinigung war freilich deutlich geworden, dass Innovation und Umsetzung neuer Ideen der ostdeutschen Wissenschaftler bei der Gestaltung der gesamtdeutschen Forschungslandschaft nicht gefragt waren. Zwar lagen solche Ideen in schriftlicher Form vor, zumal dies der Wissenschaftsrat in Vorbereitung der Evaluation auch so gefordert hatte. Aber es war bald jedem Betroffenen klar, dass die Umsetzung der Ideen und Vorschläge zur Umstrukturierung der Wissenschaftslandschaft im Osten Deutschlands aus eigener Kraft von der Politik nicht gewollt war, auch nicht solcher, die vom Wissenschaftsrat unterstützt wurden. Dies bestätigte Dieter Simon, von 1989 bis 1993 Vorsitzender des Wissenschaftsrats: »Durch die Stilisierung dieses Vorganges [also der Evaluierung und Umstrukturierung der außeruniversitären Wissenschaftslandschaft in den neuen Bundesländern - U.v.d.H.] zu einer Selbstbewertung der Wissenschaft, der sich die Politik in fraglosem Vertrauen angeschlossen hatte, wurde der erhebliche Anteil der Politik an jenen Entscheidungen wirkungsvoll verschleiert, aber deren Akzeptanz nachhaltig erhöht «. ${ }^{9}$ Noch klarer drückte das Zusammenspiel von Politik und Wissenschaftsmanagement etwa zu gleicher Zeit der damalige Präsident der LeibnizSozietät, der internationale Reputation genießende Mediziner Samuel Mitja Rapoport, aus: »Eine wahrhaft tragische Rolle war dem Wissenschaftsrat zugeteilt. Er sollte in quasi objektiver Weise die Qualität der Wissenschaft in Ost und West bewerten [...] De facto fungierte der Wissenschaftsrat als Feigenblatt für das politische Ziel, die DDR auch auf dem Gebiet der Wissenschaft zu delegitimieren «. ${ }^{10}$

Dass die Ergebnisse der Evaluation einem starken politischen Einfluss ausgesetzt waren, bestätigte auch eine Reihe von Wissenschaftlern aus den alten Bundesländern, die in der einen oder anderen Weise an der Beseitigung ostdeutscher Forschungseinrichtungen und vor allem des ostdeutschen Personals beteiligt waren. So äußerte der Philosoph Ulrich Johannes Schneider, der im Auftrag der UNESCO die Abwicklung und die allgemeine Neubesetzung philosophischer Lehrstühle in Ostdeutschland untersuchte, in einem Interview: »Die Abwicklung war vor allem ein

8 Robbe 1996, S. 194.

9 Simon 1997.

10 Bericht des Präsidenten der Leibniz-Sozietät, Samuel Mitja Rapoport, auf dem LeibnizTag am 2. Juli 1998 (Pressefassung), Bl. 4. 
politisches, nur bedingt ein wissenschaftliches Urteil über die Betroffenen, und das unter einem enormen Druck von Sachzwängen «. ${ }^{11}$

In den Empfehlungen des Wissenschaftsrats wurde unter anderem ausdrücklich die Fortsetzung der an der Akademie der Wissenschaften der DDR betriebenen Asien-, Afrika- und Orientforschung empfohlen. Denn, so hieß es dort, "gerade in Berlin sind Arbeitsgruppen entstanden, die, ungeachtet bestimmter thematischer Einschränkungen, methodischer Verkürzungen und anderer Probleme, in einer Reihe von Forschungsgebieten Neuland betreten und Beachtliches geleistet haben. Diese Arbeitsgruppen, die sich besonders mit der islamischen Welt, mit Afrika und teilweise auch mit Indien befassen, sollten dem geplanten Zentrum angehören, das sich mit den Lebensgrundlagen - jüngerer Geschichte, Sprache, Religion, Recht, Wirtschaft und Kultur - des islamischen Orients und der genannten afrikanischen Regionen befasst « ${ }^{12}$

Es sollten nach einer Evaluation der bisherigen Leistungen der ostdeutschen Geisteswissenschaftler Empfehlungen des Wissenschaftsrats erarbeitet werden, nach denen zumindest die positiv evaluierten Mitarbeiter der entsprechenden Institute der AdW der gesamtdeutschen Forschungslandschaft erhalten bleiben sollten. So schlug der Wissenschaftsrat für die positiv Evaluierten der AdW die Gründung der bereits erwähnten Förderungsgesellschaft Wissenschaftliche Neuvorhaben mbH für die Existenz von einigen Jahren vor. Die Gesellschaft mit dem ungewöhnlichen Namen war für die vom Wissenschaftsrat zuweilen mehrfach positiv evaluierten Geisteswissenschaftler der AdW unter der Schirmherrschaft der Max-Planck-Gesellschaft gegründet worden. Lange habe man im Wissenschaftsrat darüber gestritten, so der damalige Chef der VolkwagenStiftung Wilhelm Krull, »welche Form der Institutionalisierung man wählen sollte «. Träume des Westens seien hier geträumt worden. ${ }^{13}$ In zunächst acht sogenannten Forschungsschwerpunkten erhielten wissenschaftliche Mitarbeiter schließlich aus verschiedenen Akademieinstituten einen dreijährigen Arbeitsvertrag, der dann Ende 1994 noch einmal um ein weiteres Jahr verlängert wurde. Die Forschungsschwerpunkte, kurz FSP genannt, waren in der Zeit ihrer Existenz die einzigen außeruniversitären Forschungseinrichtungen auf dem Gebiet der Geisteswissenschaften in den neuen Bundesländern.

\section{Altes Personal und neue Forschungsaspekte}

Die Neugründungen sollten laut Jürgen Kocka »Forschungseinrichtungen eines neuen Typs « sein, um dem System der wissenschaftlichen Institutionen in Deutschland ein neues, zukunftsträchtiges Reformelement hinzuzufügen. ${ }^{14}$ Von ehemals 817 Geisteswissenschaftlern aus der AdW der DDR fand allerdings weniger als ein

11 Decker 1997.

12 Wissenschaftsrat 1991, S. 95.

13 Krull 2002, S. 47.

14 Kocka 1996, S. 41. 
Zehntel dort eine Anstellung. Diese Geisteswissenschaftler jedoch seien, so Dieter Simon, » unbedingt erhaltungswürdig «. ${ }^{15}$

Kocka hatte in einer Tageszeitung zuvor schon hervorgehoben, dass es in den AdW-Instituten »auch sehr viel gute und sehr gute Qualität unter den Wissenschaftlern [...] gab «. Der Wissenschaftsrat ließ keinen Zweifel daran, so hob er hervor, »daß ihre Weiterbeschäftigung erreicht werden muß, unter angemesseneren und auch für ihre Arbeit zuträglicheren Organisationsbedingungen als bisher « ${ }^{16}$

Die neuen Forschungsschwerpunkte waren indes, um einen Ausdruck des Literaturwissenschaftlers Martin Fontius zu verwenden, von »liliputanischem Ausmaß «. ${ }^{17}$ Immerhin waren an so einem FSP etwa 20 Planstellen für Wissenschaftler sowie weitere für technisches Personal - von der Sekretärin bis zur Bibliothekarin - bereitgestellt worden. Etwa die Hälfte der wissenschaftlichen Mitarbeiter des FSP Moderner Orient kam aus der Akademie der Wissenschaften, in deren Institut für Allgemeine Geschichte einer der Evaluatoren, Wolfgang Raible, immerhin einige »bunte Tupfer, häufig in Gestalt jüngerer Wissenschaftler ", glaubte ausfindig gemacht zu haben, die in »interessanten Abteilungen z. B. für afrikanische Geschichte oder für moderne Orientforschung arbeiteten «. ${ }^{18}$

In der Tat war etwa die Hälfte des FSP-Personals ausdrücklich für Mitarbeiter aus der DDR-Akademie vorbehalten gewesen, und diese wurden somit zur »Gründergeneration « der neuartigen gesamtdeutschen Forschungsinstitutionen. Wie in dieser für moderne Orientforschung wurden auch die anderen aufgrund der Wissenschaftsrats-Empfehlungen geschaffenen Forschungsinstitute mit neu eingestellten Kollegen aus den alten Bundesländern »durchmischt«. Unter dem neudeutschen Begriff »Durchmischung " wurde der Vorgang verstanden, dass sich auf die noch freien Personalstellen, also pro FSP etwa zehn, Wissenschaftler von außerhalb der AdW bewerben konnten. Somit war gut die Hälfte des Personals in den auch für die alte Bundesrepublik neuartigen außeruniversitären Forschungseinrichtungen mit Ost-, die andere Hälfte mit Westwissenschaftlern besetzt.

Die aus dem Akademie-Institut für Allgemeine Geschichte in den FSP übernommenen Projekte basierten zum überwiegenden Teil auf Vorarbeiten der betreffenden Wissenschaftler. Sie wurden in das Forschungsprogramm der neuen Institution integriert, nämlich unter der etwas weit gefassten Überschrift »Beziehungsgeschichtliche Fragestellungen des Verhältnisses zwischen Orient und Okzident « ${ }^{19}$ Unter dieser Themenstellung wurden Forschungen fortgeführt, die in der DDR Tradition hatten und die im internationalen Kontext sinnvoll schienen.

Gegliedert war das Forschungsvorhaben in zwei Hauptgruppen, in die sich jeder Mitarbeiter einbringen sollte. Es wurden in den Gruppen die Themen »Indigene

15 Simon 1996, S. 26.

16 Kocka 1991.

17 Fontius 1991, S. 38.

18 Raible 1992, S. 58 f.

19 Förderungsgesellschaft Wissenschaftliche Neuvorhaben 1993, S. 93. 
Transformation als Prozess. Auseinandersetzung, Synthese und Neubildung « 20 sowie "Wechselseitigkeit in ungleichen Beziehungen: Deutschland, der Orient und Afrika im 18. bis 20. Jahrhundert « in mehreren Teilprojekten bearbeitet. ${ }^{21}$ »Bei aller Ungleichheit der Beziehungen zwischen Orient und Okzident gehen wir davon aus, daß sich die Kulturen gegenseitig beeinflußt haben. Und dabei interessieren uns langfristige Entwicklungen und deren Analyse ", erklärte der kommissarische Leiter des FSP Moderner Orient zu den inhaltlichen Zielvorstellungen der Projekte in einem Zeitungsinterview. ${ }^{22}$

Der neu berufene Wissenschaftliche Beirat des FSP, dem führende Experten ihrer Fachgebiete aus der Bundesrepublik angehörten, die die Orientalistik, Asienwissenschaften und Afrikawissenschaften vertraten, bestätigte am 6. April 1992 die beiden genannten inhaltlichen Forschungslinien, die gleichberechtigt auf die regionalen Schwerpunkte Naher Osten, Afrika und Südasien verteilt worden waren. ${ }^{23}$ Einige Mitglieder dieses Beirats hatten schon am Evaluierungsprozess der Ex-DDR-Wissenschaft teilgenommen und galten somit als Kenner der Stärken und Schwächen ihrer Kollegen aus dem Osten Deutschlands.

Im ersten Tätigkeitsbericht des FSP Moderner Orient aus dem Jahre 1993 wurde mitgeteilt, dass sich eine Vertiefung und Verbreitung in den "Diskussions- und Kooperationsebenen « ergeben habe, so beispielsweise in dem Projektschwerpunkt "Missionsgeschichte als Quelle für Beziehungs- und Sozialgeschichte «24, in dem verschiedene Einzelprojekte aus den Bereichen der Afrika- und Asienforschung eingegangen waren. Auch in anderen Forschungsgruppen des FSP war man mit Elan ans Werk gegangen, hatten sich doch die Arbeitsbedingungen im Vergleich zu DDRZeiten erheblich verbessert. Eine Bibliothek wurde aufgebaut, die unter anderem einen deutlichen Schwerpunkt auf das südliche und östliche Afrika legte.

Die ursprünglich zehn Personalstellen für den FSP Moderner Orient wurden später im Verlauf der "Durchmischung " auf das Doppelte erhöht. Schließlich arbeiteten bis 1994 neben zirka 15 Asien- sowie Orientwissenschaftlern sechs Afrikawissenschaftler am FSP Moderner Orient. ${ }^{25}$ Schon zu diesem Zeitpunkt stimmte jedoch die »Durchmischung « im Verhältnis eins zu eins nicht mehr: Bis auf eine Ausnahme kamen nämlich schon nach knapp zwei Jahren alle Afrikawissenschaftler des FSP Moderner Orient aus den alten Bundesländern. In den anderen Regionalbereichen sah es nicht viel anders aus. Für die in die Arbeitslosigkeit entlassenen oder für die in kurzzeitigen öffentlich geförderten Arbeitsmaßnahmen gedrängten Wissenschafter der AdW gab es kein Zurück mehr an die wissenschaftlichen Institutionen, so

20 Vgl. zu dieser Thematik ausführlicher Forschungsschwerpunkt Moderner Orient der Förderungsgesellschaft Wissenschaftliche Neuvorhaben 1994, S. 741 ff.

21 Vgl. ausführlicher Förderungsgesellschaft Wissenschaftliche Neuvorhaben 1993, S. 94 ff.

22 Zitiert in Böhme 1995.

23 Vgl. Schwanitz 1993, S. 58.

24 Förderungsgesellschaft Wissenschaftliche Neuvorhaben 1994, S. 127.

25 Vgl. Arbeitskreis der Deutschen Afrika-Forschungs- und Dokumentationsstellen 1994, S. $19 \mathrm{ff}$. 
sehr einzelne von ihnen sich auch durch die Erarbeitung von akademisch durchaus beachtenswerten Forschungsleistungen dafür ausweisen konnten und internationale Anerkennung für ihre Publikationen erlangten. Es entstand auf diese Weise der sogenannte zweite Wissenschaftsmarkt im Osten Deutschlands.

Die Arbeit der Forschungsschwerpunkte der Förderungsgesellschaft Wissenschaftliche Neuvorhaben wurde von deren Geschäftsführung, von der Max-PlanckGesellschaft und auch von den Mitarbeiterinnen und Mitarbeitern nach drei bis vier Jahren anstrengender Aufbauarbeit als erfolgreich eingeschätzt. Beurteilt oder evaluiert wurden die einzelnen Arbeitsleistungen allerdings nicht. Es kann jedoch davon ausgegangen werden, dass die in der DDR bereits begonnenen Forschungsthemen erfolgreich weiterbearbeitet und innovativ weiterentwickelt worden sind. ${ }^{26}$ In verschiedenen Publikationen und in Politikerreden wurden deshalb die FSP als Beweise des erfolgreichen Umbaus der ostdeutschen Wissenschaftslandschaft bezeichnet.

\section{Die Nichteinhaltung der Empfehlungen des Wissenschaftsrats am Beispiel des FSP Moderner Orient}

Im letzten Jahr der ursprünglich vorgesehenen dreijährigen Existenz des FSP Moderner Orient, 1994, arbeiteten neben Wissenschaftlern auch zeitweilig einige Doktoranden. ${ }^{27}$ Um ein Jahr, also für das Jahr 1995, wurde die Existenz des FSP und somit auch die Arbeitsverträge der dort Beschäftigten verlängert. In den letztlich insgesamt vier Jahren Aufbauarbeit des FSP Moderner Orient herrschte nach allem, was bekannt geworden ist, zwischen den Wissenschaftlern aus Ost und West eine innovationsfreundliche Atmosphäre, zumal die Mitarbeiter in der Regel der jüngeren Generation angehörten.

Die intensive Arbeitsphase des Auf- und Ausbaus der außeruniversitären Forschungszentren und das gegenseitige Kennenlernen von Ost- und Westwissenschaftlern wurden von der Geschäftsführung der Förderungsgesellschaft, von der zuständigen Berliner Senatsverwaltung, von den Verantwortlichen der Max-PlanckGesellschaft und auch von den Mitarbeiterinnen und Mitarbeitern selbst als fruchtbar eingeschätzt. Nicht nur im Aufbau neuer Organisationsstrukturen zeigte sich das Engagement eines jeden Einzelnen, sondern auch in den internationale Anerkennung findenden Forschungsergebnissen.

Die in den FSP erbrachten Arbeitsleistungen bildeten jedoch keine Basis für eine Weiterbeschäftigung der dort Aufbauarbeit leistenden Wissenschaftler. ${ }^{28}$ Vielmehr wurden die begonnenen oder geplanten Projekte 1995 abrupt abgebrochen. Denn am 31. Dezember 1995 gab die Max-Planck-Gesellschaft ihre, nennen wir es, Fürsorgepflicht für die aus dem Osten Deutschland stammenden Wissenschaftler und die nicht zuletzt mit deren Engagement aufgebauten Forschungsschwerpunkte ab,

26 Vgl. Krauth, Wolz 1998; Kocka, Mayntz 1998.

27 Vgl. Arbeitskreis der Deutschen Afrika-Forschungs- und Dokumentationsstellen 1994, S. $19 \mathrm{ff}$.

28 Vgl. Krauth, Wolz 1998. 
und das alte, in der bundesrepublikanischen Wissenschaftslandschaft übliche »DFG-Projekte-Prinzip« kam zur Geltung.

\section{Das Ende der Fürsorgepflicht}

Die erreichten Erfolge, die ja für jeden, der sich dafür interessiert hätte, sicht- und abrechenbar gewesen wären, sollten für die Verantwortlichen in ihrer Entscheidung zum Übergang der Zuständigkeit der FSP von der Max-Planck-Gesellschaft an die Deutsche Forschungsgemeinschaft (DFG) nicht ins Gewicht fallen. Nach dem Auslaufen der "Schirmherrschaft " der MPG für die ostdeutschen Wissenschaftler Mitte der 1990er Jahre wurden die FSP, vor allem jedoch der FSP Moderner Orient, von in der DDR sozialisierten Wissenschaftlern mehr oder weniger rigoros "gesäubert $\ll{ }^{29}$

Zum Schaden kam für die Betroffenen auch noch Spott hinzu, als im Nachhinein Stimmen von anonym gebliebenen führenden Wissenschaftlern beziehungsweise Wissenschaftsmanagern der Bundesrepublik hörbar wurden, dass jene »doch gleich hätten sehen müssen, daß das Ganze schiefgehen würde, und sich beizeiten nach einer anderer Tätigkeit " umsehen können. ${ }^{30}$ Bei solch einer Vorgehensweise liegt die Vermutung nahe, dass man »uns einfach weg vom Fenster haben [will]. Aus Konkurrenz-, vielleicht auch aus anderen Gründen «31, wie es der Sprecher einer dem Personalrat der Humboldt-Universität angeschlossenen Kommission Anfang Oktober 1996 in einem Interview in Bezug auf ostdeutsche Geisteswissenschaftler äußerte.

Zu Beginn des Jahres 1996 wurden die FSP als Institution - wohlgemerkt: nicht mit dem bisherigen Personal - in ein » Geisteswissenschaftliche Zentren « überführt, die in drei der neuen Bundesländer stationiert wurden. So konnte immerhin ausgeschlossen werden, dass »das einzige Reformunternehmen [...], das im Bereich Geisteswissenschaften aus der Vereinigung beider Wissenschaftssysteme hervorgegangen ist «, zu Grabe getragen werden musste, ${ }^{32}$ wie es der damalige stellvertretende SPD-Vorsitzende und Vize-Bundestagspräsident Wolfgang Thierse einmal formulierte.

Aus dem FSP Moderner Orient wurde nunmehr das Geisteswissenschaftliche Zentrum Moderner Orient, das kaum noch Mitglieder der "Gründergeneration " beschäftigte - ein Schicksal, das auch einige westdeutsche Kollegen traf, die am FSP Aufbauarbeit geleistet hatten. Ob die vollständige Liquidierung der FSP einer Option der herrschenden politischen Parteien entsprach, mit der man die noch immer als ehemalige Gegner aus dem Kalten Krieg betrachteten »Ossis « loswerden

29 Vgl. zum genauen Hergang und den Hintergründen sowie den Methoden der Verdrängung der aus der AdW stammenden Forscher die Untersuchung von van der Heyden 2002, S. 113-154.

30 Zitiert in Zimmer 1996.

31 Sager 1996.

32 Thierse 1994.

Leviathan, 41. Jg., 4/2013 
wollte? Was auch immer an Motiven dahinter stand, die »Hülle", die Institution, die Bibliothek, die Ausstattung, das Logo, die Unterkunft und einiges andere konnten so unter dem Dach eines neu gegründeten Vereins erhalten bleiben.

In der Internetpräsentation jenes »Vereins Geisteswissenschaftliche Zentren Berlin e.V. « liest sich dessen Institutionsgeschichte, dabei die Kontinuitäten - allerdings bei Nichtnennung der Gründe für die erwähnten Empfehlungen des Wissenschaftsrats - nicht verschweigend, wie folgt: Auf Grundlage der Empfehlungen des Wissenschaftsrats vom Juni 1991 und vom November 1994 initiierte der Wissenschaftssenator im Land Berlin Ende 1995 die Gründung des Vereins Geisteswissenschaftliche Zentren Berlin e.V. und übertrug ihm die Trägerschaft für drei Forschungszentren: Zentrum für Allgemeine Sprachwissenschaft (ZAS), Zentrum für Literatur- und Kulturforschung (ZfL) und das Zentrum Moderner Orient (ZMO).

Am 1. Januar 1996 nahmen die drei Zentren - nach vierjährigem Vorlauf in der von der MPG eingerichteten und betreuten Fördergesellschaft Wissenschaftliche Neuvorhaben mbH - ihre Tätigkeit auf. Die aktuelle Förderung seit 1. Januar 2008 durch das Land Berlin in der Grundausstattung, durch das Bundesministerium für Bildung und Forschung für drei Forschungsprogramme in der Ergänzungsausstattung sowie durch eine Reihe von Einzelbewilligungen nationaler und internationaler Fördereinrichtungen basiert auf einer Empfehlung des Wissenschaftsrats vom 30. Januar 2006.33

Kaum die Hälfte ehemaliger AdW-Mitarbeiter wurde in der neu firmierten Forschungsinstitution zumeist befristet weiterbeschäftigt. Ein allzu rigoroser Schnitt hätte dem Image der Bundesrepublik sicherlich geschadet. Denn ein Ende der außeruniversitären Forschungszentren hätte für die neuen Bundesländer bedeutet, dass das dortige geisteswissenschaftliche Potenzial, wie es der damalige DFG-Präsident Wolfgang Frühwald errechnete, auf unter vier Prozent des früheren Umfangs gesunken wäre. ${ }^{34}$ Die Bundesrepublik brauchte sich jedoch dieses blamable Zeugnis nicht auszustellen, denn die FSP blieben ja als Institutionen, wenn auch nunmehr unter einem leicht veränderten Namen, erhalten - allerdings ihres ursprünglichen politischen Auftrags, die Weiterbeschäftigung von AdW-Mitarbeitern zu garantieren, beraubt.

Für die Betroffenen war diese Handlungsweise vor allem deshalb unbegreiflich, weil die Umwandlung vom FSP zum Geisteswissenschaftlichen Zentrum Moderner Orient den von Vertretern der Max-Planck-Gesellschaft und anderen wissenschaftlichen und politischen Institutionen zuvor immer wieder geäußerten Versprechungen widersprach. Zugesichert war eine Evaluation der bisherigen erbrachten Leistungen in den vergangenen vier Jahren harter Arbeit, in denen - das kann wohl gesagt werden - die Forschungsschwerpunkte sich in der internationalen wissenschaftlichen Gemeinschaft verankert hatten. Tatsächlich spielten die Ergebnisse in Form von Publikationen, durchgeführten Konferenzen, eingeladenen Gastwissenschaftlern, Drittmitteleinwerbungen, verabredeten oder begonnenen nationalen und

33 Vgl. www.gwz-berlin.de/ueber-die-gwz/organisation (Zugriff 29.09.2013).

34 Vgl. Tätigkeitsbericht des Gesamtbetriebsrats der Max-Planck-Gesellschaft, Betriebsräteversammlung 1994, ohne Ort, S. 21. 
internationalen Kooperationsprojekten, Öffentlichkeitsarbeit und ähnliche Aktivitäten nun aber überhaupt keine Rolle mehr: Alle bisherigen Mitarbeiter hatten sich mit neuen Projekten bei der DFG zu bewerben.

Die Begutachtung geplanter Projekte war zwar übliche Praxis in der bundesdeutschen Wissenschaftsförderung. Diese hätte, so könnte man argumentieren, auch für die ehemaligen Akademiemitarbeiter und die »Gründungsmitglieder « der FSP gelten sollen. Jedoch wird dabei die Tatsache missachtet, dass es sich um besondere Institutionen handelte, die in der gesamtdeutschen Wissenschaftslandschaft neuen und bislang einzigartigen Institutionen der FSP aus dem Altbestand der DDR-Wissenschaftslandschaft neu entstandene Institutionen gewesen sind und aufgrund der positiv evaluierten Ost-Wissenschaftler überhaupt erst entstanden waren.

Das Modell der FSP und seiner Nachfolgeeinrichtungen war das Ergebnis der Abwicklung vornehmlich der außeruniversitären geisteswissenschaftlichen Forschungseinrichtungen der DDR sowie des Bemühens einiger perspektivisch denkender Wissenschaftsmanager um den damaligen Wissenschaftsratsvorsitzenden Dieter Simon und von Jürgen Kocka, der sich um eine objektive Bewertung der ostdeutschen Geisteswissenschaften verdient gemacht hat. Ziel war es, Erhaltenswertes zu bewahren. ${ }^{35}$ Mit dem Übergang vom FSP zum GWZ war diese Absicht jedoch für die Afrika-, Orient- und Asienforschung aufgegeben worden.

\section{Fragen und keine Antworten}

Einige Fragen stehen bei der Bewertung dieser kurz skizzierten Entwicklungen im Raum: Wie war es möglich, dass nach nur wenigen Jahren die ehemals definierten Aufgaben einer Institution so umgewidmet wurden, dass von den ursprünglichen Intentionen und schriftlich niedergelegten Empfehlungen des höchsten wissenschaftlichen Beratungsorgans der Bundesrepublik nichts übrig blieb? Wieso konnte so gut wie ohne Protest der deutsche Einigungsvertrag, der ja den Erhalt positiv evaluierter Wissenschaftler aus der DDR für die gesamtdeutsche Wissenschaftslandschaft vorsah, gebrochen werden?

Was war konkret geschehen? Die Gutachter der DFG hatten entschieden, die Nachfolgeinstitution des FSP Moderner Orient personell etwa um ein Drittel zu verkleinern. Dies löste nicht nur bei den nunmehr um ihre wissenschaftliche Zukunft Betrogenen Betroffenheit aus. Immerhin war von der Reduzierung des Personalbestands des FSP Moderner Orient vor allen anderen FSP am stärksten betroffen. Aus der AdW übernommene Forschungsvorhaben und Methoden verschwanden. Die Enttäuschung war auch deshalb groß, weil in der Vergangenheit etliche Projekte in internationalen Dimensionen und Kooperationen angeregt, durchgeführt, diskutiert und angeschoben worden waren, die nun ein überraschend sinnloses Ende fanden. ${ }^{36}$

35 Vgl. Krauth 1998, S. $21 \mathrm{ff}$.

36 Vgl. die Tätigkeitsberichte der Förderungsgesellschaft Wissenschaftliche Neuvorhaben von 1992, 1993 und 1994. 
Besonders enttäuschend war das Ergebnis des DFG-Gutachterverfahrens für die aus der AdW kommenden Wissenschaftler, hatten sie doch zumindest partiell den Empfehlungen des Wissenschaftsrats vertraut. Seit Gründung des FSP waren ihnen von einigen Entscheidungsträgern immer wieder Versprechungen gemacht worden, etwa dahingehend, dass es für durch das DFG-Gutachterverfahren nicht übernommene Mitarbeiter aus der AdW noch andere Lösungen geben werde, dass es sicherlich in der ersten Zeit für die negativ Evaluierten Anschubfinanzierungen geben werde, dass man die Verantwortung zumindest gegenüber den ehemaligen Akademie-Mitarbeitern nicht abgeben wolle, dass man eine Quotierung von Ost- und Westwissenschaftlern anstreben könnte, dass das Profil des FSP in seiner Kombination von Ost- und Westwissenschaftlern sich bewährt habe und beibehalten werden solle, und ohnehin gäbe es die Option der sogenannten kw-Stellen, das heißt, dass die vorhandenen Personalstellen bis zum Ausscheiden der sie besetzenden Wissenschaftler weiter finanziert werden, bis diese aus Alters- oder sonstigen Gründen ausgeschieden sind. Außerdem machte die Mitteilung Hoffnung, dass die MaxPlanck-Gesellschaft mit der neuen Form der Förderung außeruniversitärer Forschungsinstitutionen ja immerhin Neuland beschritten habe und demzufolge auch auf neue Weise die Begutachtung und Rekrutierung der Mitarbeiter vornehmen würde. Im Nachhinein jedoch lässt sich der Eindruck nicht verhehlen, dass die Versprechungen einzelner Vertreter der für die Umsetzung der Empfehlungen des Wissenschaftsrats Verantwortlichen einzig und allein dem Ziel hatten dienen sollen, »Ruhe« und ein Gefühl der Sicherheit bei den Betroffenen zu erzielen.

Ursprünglich wurde für die FSP-Mitarbeiter die Parole ausgegeben, dass sich jeder mit einem eigenen Projekt bei der DFG zu bewerben habe, da es nunmehr mit der Abgabe der Verantwortung der MPG für die Forschungsschwerpunkte keine kollektive Förderung mehr geben werde. Der Vorteil sollte im Vergleich zu den ansonsten üblichen "Normalverfahren « der DFG darin bestehen, dass auch »einfache « Wissenschaftler ohne Vermittlung eines Lehrstuhlinhabers einen Antrag auf Sachund Personalmittelförderung stellen durften. Dann hieß es plötzlich, dass alle individuellen Anträge unter einem einheitlichen »inhaltlichen Dach « miteinander verbunden sein sollten. Das sollte - so hieß es beschwichtigend - die Chancen des Einzelnen erhöhen. Tatsächlich hatte der von der DFG geforderte einheitliche Gesamtantrag des FSP - an dem sich alle Mitarbeiter und Mitarbeiterinnen der drei Regionalbereiche Asien, Afrika und Orient - beteiligen sollten, im Ergebnis sogar vergleichsweise schlechtere Erfolgschancen für die einzelnen Antragsteller als ein Antrag im Normalverfahren. Denn trotz der von der DFG im Antragsstellungsprozess immer wieder betonten Notwendigkeit einer Kohärenz der Anträge, die zwangsläufig zu gewissen Verknappungen bei der Argumentation in den Einzelanträgen führten, wurden dann durch die sieben Gutachter - für die Betroffenen selbstverständlich überraschend - doch lediglich die Einzelanträge begutachtet und einige von ihnen als nicht förderungswürdig eingestuft.

Um gut ein Drittel des Personalbestands durch die Empfehlungen der Evaluierungskommission der DFG personell geschwächt, fand das Gesamtunternehmen FSP Moderner Orient dann doch noch die Gnade der Gutachter, die für einen Fortbestand in dieser verkleinerten Form plädierten. Vielleicht ist ein Beleg für die 
»Objektivität « des scharfen Vorgehens der Gutachter dadurch gegeben, wenn man darauf verweist, dass einige abgelehnte Anträge dann später, wenn sie von einer Universität aus eingereicht wurden, von der DFG bewilligt worden sind. Der Wissenschaftsjournalist Paul Stoop bot im » Tagesspiegel « folgende Erklärung an: » Gutachter, die selbst in der Regel von den Universitäten kommen, wo Überspezialisierung und Isolierung die Regel sind, entscheiden über das Schicksal von Projekten, die gerade diese Fehler vermeiden sollen und in einem größeren Zusammenhang erarbeitet werden «. 37

Auch Dieter Simon sprach in diesem Zusammenhang von »einem langen Leidensweg zusehends strangulierter 'geisteswissenschaftlicher Zentren« «, die nunmehr » unter weiter steigenden personellen Verlusten bei der DFG als interuniversitäre Bittsteller-Vereine ums Überleben kämpfen « müssen. ${ }^{38}$

Weniger als die Hälfte des ursprünglichen wissenschaftlichen FSP-Personals durfte ab 1996 auf ihrem Arbeitsplatz - in einer Institution, die für sie geschaffen worden war, die sie aufgebaut und getragen hatten und die nun Geisteswissenschaftliches Zentrum Moderner Orient hieß - weiterarbeiten. Eine hilfreiche Hand des neuen Zentrums wurde den nun arbeitslos dastehenden Kolleginnen und Kollegen nicht gereicht. So gelangte das Institut für Hochschulforschung in Halle-Wittenberg, welches im Auftrag der Berliner Senatsverwaltung für Wissenschaft und Forschung eine Studie über die Ostberliner Wissenschaftslandschaft vorgelegt hatte, im Jahre 2004 zu der Einschätzung, dass der zweite Gründungsauftrag der Geisteswissenschaftlichen Zentren, nämlich die Integration von ehemaligem AkademiePersonal, nicht erfüllt worden ist. Laut Auskunft des Vorstands der Geisteswissenschaftlichen Zentren e.V. jedoch habe sich das Thema unterdessen ohnehin erledigt, »da mit den bestehenden GWZ eine erfolgreiche Integration zwischen den Wissenskulturen von Ost und West erreicht wurde. ${ }^{39}$ Dass diese Art der »Integration « dann eher als eine Verhöhnung der ostdeutschen Geisteswissenschaftler von der AdW empfunden wurde, von denen zu jenem Zeitpunkt kaum noch jemand, zumindest nicht im GWZ Moderner Orient, arbeitete, überrascht nicht.

\section{8. »Feine Unterschiede« - auch in der Berliner »Orientforschung «}

Der französische Soziologe Pierre Bourdieu ${ }^{40}$ spricht in Bezug auf die Ausgrenzung einer bestimmten Gruppierung von Menschen aus einer eigentlich gemeinsam zu bewerkstelligenden Aufgabe von der Distanz der » feinen Unterschiede «. Der Begriff meint die Tendenz gesellschaftlicher Schichten, sich gegeneinander abzugrenzen. Vor allem Eliten reproduzieren sich am liebsten aus den eigenen Reihen. ${ }^{41}$

37 Stoop 1996.

38 Simon 1996, S. 25.

39 Zitiert in Bloch, Pasternack 2004, S. 46.

40 Bourdieu 1987.

41 Vgl. dazu etwa den Titel einer Sendung im Deutschlandradio vom Oktober 2012: »Wessis stellen Wessis ein".

Leviathan, 41. Jg., 4/2013 
Mit Stand Dezember 2002 waren von 141 Mitarbeiterinnen und Mitarbeitern aller Berliner Geisteswissenschaftlichen Zentren lediglich 15 bereits in der AdW beschäftigt gewesen. ${ }^{42}$ Von den seit 1996 neu eingestellten Mitarbeiter wies nur eine Minderheit eine ostdeutsche Sozialisation auf.

Die anhaltende Verdrängung der ostdeutschen Wissenschaftler aus dem gesamtdeutschen Wissenschaftsbetrieb wurde bereits vor einigen Jahren von renommierten internationalen Wissenschaftlern mit Sorge betrachtet. In einer Anzeige in der »Frankfurter Rundschau" vom September 2002 schrieben unter anderem Eric Hobsbawm, Friedrich Katz und Wilma und Georg Iggers: »Wir, die Unterzeichner, mit der deutschen Kultur und Wissenschaft verbundene Forscher und Universitätslehrer, wenden uns an die Regierung der Bundesrepublik Deutschland und an Verantwortliche für Wissenschaftspolitik in den deutschen Bundesländern, um diese zu schnellstmöglicher Hilfeleistung im Interesse der wenigen, sich noch in der deutschen Wissenschaftslandschaft [...] behauptenden Forscherinnen und Forscher der früheren DDR zu veranlassen. Elf Jahre nach der staatlichen, von der deutschen Bevölkerung mehrheitlich gewünschten, aber auf gleichberechtigter Basis erhofften Vereinigung des Landes ist die intellektuelle 'Infrastruktur in Ost- und Westdeutschland unterschiedlicher, als sie es je in der Zeit der Teilung war. [...] Ohne ostdeutsche Intellektuelle in qualifizierten Berufen kann [...] die in der früheren DDR sich ausbreitende Enttäuschung über den als Anschluss empfundenen Prozess der Überstülpung westdeutscher Strukturen und Denkweisen [...] nicht überwunden werden $« .43$

Dieser Aufruf blieb allerdings ungehört. Das GWZ Moderner Orient ist in der Zwischenzeit zu einer anerkannten geisteswissenschaftlichen Institution in Deutschland und in der Welt geworden. Doch sollte man nicht vergessen, dass es sich um eine Einrichtung handelt, deren Fundamente von vielen damals zumeist jungen Wissenschaftlern aus Ost- und Westdeutschland in der ersten Hälfte der 1990er Jahre gelegt wurden, die danach zum Teil wieder entlassen wurden.

Und es sollten auch nicht die Fundamente der Fundamente vergessen werden, nämlich die Kolleginnen und Kollegen aus der Akademie der Wissenschaften, die wie es im Jahr 2012 der Bremer Jurist und Philosoph Hans Jörg Sandkühler in einem Interview nannte - aus »Unkenntnis « und aufgrund von »Vorurteilen « der Evaluatoren ihres Arbeitsplatzes und ihrer beruflichen Existenz beraubt wurden. ${ }^{44}$ Es ist freilich kaum verwunderlich, dass die betroffenen ehemaligen Mitarbeiter der Akademie der Wissenschaften die 20-jährige Geschichte des FSP beziehungsweise des GWZ Moderner Orient mit wenig Euphorie sehen, weil ihre geleistete, ehemals von den westdeutschen Kollegen »(ab)geteilt $(\mathrm{e})$ « Arbeit zwar durch eine Evaluation durchaus gewürdigt und dann »vereint " mit den Kollegen fortgeführt werden konnte, ihre Protagonisten jedoch nach der Etablierung einer eigentlich zukunftsweisenden Institution vom "globalen « Forschen ausgeschlossen wurden.

42 Vgl. Bloch, Pasternack 2004, S. 46.

43 Anzeige in der Frankfurter Rundschau vom 11. September 2002.

44 Vgl. Sandkühler 2012. 


\section{Literatur}

Arbeitskreis der Deutschen Afrika-Forschungs- und Dokumentationsstellen 1994. Rundbrief 1/2. Hamburg.

Bloch, Roland; Pasternack, Peer 2004. Die Ost-Berliner Wissenschaft im vereinigten Berlin. Eine Transformationsfolgenanalyse. HoF-Arbeitsbericht 2, hrsg. v. HoF Wittenberg - Institut für Hochschulforschung an der Martin-Luther-Universität Halle-Wittenberg.

Böhme, Christian 1995. »Katecheten zwischen den Kulturen. Die künftigen geisteswissenschaftlichen Zentren (4): Der Forschungsschwerpunkt Moderner Orient ", in Der Tagesspiegel, 28. Februar 1995.

Bourdieu, Pierre 1987. Die feinen Unterschiede. Kritik der gesellschaftlichen Urteilskraft. Frankfurt a. M.: Suhrkamp.

Decker, Kerstin 1997. »Wenn Philosophen sich streiten. Gab es in der DDR nur eine staatlich verordnete Philosophie oder auch philosophisches Denken? ", in Der Tagesspiegel, 12. August 1997.

Fontius, Martin 1991. "Der Akademie zum Abschied «, in Mitteilungen des deutschen Germanistenverbandes 38, 2, S. 37-60.

Förderungsgesellschaft Wissenschaftliche Neuvorhaben mbH. Hrsg. 1993. Tätigkeitsbericht 1992 der geisteswissenschaftlichen Forschungsschwerpunkte. München: Biering.

Förderungsgesellschaft Wissenschaftliche Neuvorhaben mbH. Hrsg. 1994 Tätigkeitsbericht 1993 der geisteswissenschaftlichen Forschungsschwerpunkte. München: Biering.

Förderungsgesellschaft Wissenschaftliche Neuvorhaben mbH. Hrsg. 1995 Tätigkeitsbericht 1994 der geisteswissenschaftlichen Forschungsschwerpunkte. München: Biering.

Forschungsschwerpunkt Moderner Orient der Förderungsgesellschaft Wissenschaftliche Neuvorhaben mbH 1994. Jabrbuch 1993, hrsg. von der Generalverwaltung der Max-PlanckGesellschaft München. Göttingen: Vandenhoeck \& Ruprecht.

Frühwald, Wolfgang et al. 1990. Geisteswissenschaften heute. Eine Denkschrift. Konstanz: Universität Konstanz.

Heyden, Ulrich van der 2002. »Wie die Afrikawissenschaft in Ostdeutschland durch eine sspäte Abwicklung < beseitigt wurde", in Deutsche Einheit und Elitenwechsel in Ostdeutschland, hrsg. v. Bollinger, Stefan; Heyden, Ulrich van der, S. 113-154. Berlin: trafo-Verlag.

Kocka, Jürgen 1991. »Ein Gutachter erwidert den Evaluierten ", in Der Tagesspiegel, 12. September 1991.

Kocka, Jürgen 1996. »Universitäre und außeruniversitäre Forschung. Ein zentrales Problem der Reform ", in Die Zukunft der Geistes- und Sozialwissenschaften in Ländern Mitteleuropas unter Berücksichtigung von Erfahrungen aus dem deutschen Einigungsprozeß. Symposium Halle (Saale), 1.-4. November 1995, hrsg. v. Berberich, Thomas, S. 35-43. Bonn, Washington: Deutsch-Amerikanisches Konzil.

Kocka, Jürgen; Mayntz, Renate. Hrsg. 1998. Wissenschaft und Wiedervereinigung. Disziplinen im Umbruch. Berlin: Akademie Verlag.

Krauth, Wolf-Hagen 1998. »Asien- und Afrikawissenschaften ", in Wissenschaft und Wiedervereinigung. Disziplinen im Umbruch, hrsg. v. Kocka, Jürgen; Mayntz, Renate, S. 21-78. Berlin: Akademie Verlag.

Krauth, Wolf-Hagen; Wolz, Ralf. Hrsg. 1998. Wissenschaft und Wiedervereinigung. Asien- und Afrikawissenschaften im Umbruch. Berlin: Akademie-Verlag.

Krull, Wilhelm 2002. »Die Wiedervereinigung vorausdenken. Die Neugestaltung der außeruniversitären Forschungseinrichtungen. Prinzipien und Ergebnisse ", in 10 Jahre danach. Zur Entwicklung der Hochschulen und Forschungseinrichtungen in den neuen Ländern und Berlin, hrsg. v. Stifterverband für die Deutsche Wissenschaft, S. 44-47. Essen: Stifterverband für die Deutsche Wissenschaft.

Raible, Wolfgang 1992. "Impressionen beim Evaluieren. Zur Abwicklung der kulturwissenschaftlichen Einrichtungen der ehemaligen DDR-Akademie der Wissenschaften ", in Die Abwicklung der DDR, hrsg. v. Arnold, Heinz-Ludwig; Meyer-Gosau, Frauke, S. 54-63. Göttingen: Wallstein.

Robbe, Martin 1996. "Die Asien- und Afrikawissenschaften im Umbruch. Tagung der BerlinBrandenburgischen Akademie der Wissenschaften am 4./5. Oktober 1995. Ein kommentierender Bericht", in Asien - Afrika - Lateinamerika 22, 2, S. 194.

Leviathan, 41. Jg., 4/2013 
Sager, Dietmar 1996. "Kippt die Politik WIP? «, in Neues Deutschland, 4. Oktober 1996.

Sandkühler, Hans Jörg 2012. »Philosophen als Politberater. Interview mit Hans Jörg Sandkühler «, in Neues Deutschland, 15. November 2012.

Schattenfroh, Silvia 1998. "Biomedizin in Ostdeutschland vor und nach der Wende", in Deutsche Medizinische Wochenschrift 13, 27. März 1998.

Schwanitz, Wolfgang 1993. »Vereint entzweit. Deutsche Akademiker zwischen Zerschlagung und Einpassung aus der Sicht eines Berliner Orientalisten 1986-1992 «, in Berliner Debatte INITIAL. Zeitschrift für sozialwissenschaftlichen Diskurs 4, S. 54-64.

Simon, Dieter 1995. "Verschleudert und verschludert. Die Wissenschaftsruinen des Westens waren das Vorbild für die Reform im deutschen Osten. Sie wurden dankbar angenommen. Mittelmaß und Anpassung sind das Ergebnis. Ein Mittäter zieht selbstkritisch Bilanz «, in Die Zeit, 7. April 1995.

Simon, Dieter 1996. »Die Gesellschaftswissenschaften nach der deutschen Einigung ", in Die Zukunft der Geistes- und Sozialwissenschaften in Ländern Mitteleuropas unter Berücksichtigung von Erfahrungen aus dem deutschen Einigungsprozeß. Symposium Halle (Saale), 1.-4. November 1995, hrsg. v. Berberich, Thomas, S. 26-34. Bonn, Washington: DeutschAmerikanisches Konzil.

Simon, Dieter 1997. »Im Block. Ein Vorsitzender blickt auf seine vielfältigen Erfahrungen zurück: Wie kluge Köpfe sich in raffinierten Kompromissen einzimmern ", in Die Zeit, 27. September 1997.

Stoop, Paul 1996. »Bescheiden, aber optimistisch in die Zukunft. Die Geisteswissenschaftlichen Zentren mußten Federn lassen. Begutachtung durch die DFG abgeschlossen ", in Der Tagesspiegel, 12. September 1996.

Thierse, Wolfgang 1994. Geisteswissenschaftliche Forschungsschwerpunkte in Ostdeutschland erhalten und sichern. Pressemitteilung der SPD-Bundestagsfraktion, Bonn, 4. November 1994.

Wissenschaftsrat. Hrsg. 1991. Stellungnahme zu den außeruniversitären Forschungseinrichtungen der ehemaligen Akademie der Wissenschaften der DDR auf dem Gebiet der Geisteswissenschaften. Düsseldorf, 5. Juli 1991.

Zimmer, Dieter E. 1996. »Amtlich betrogen. Wie die Bundesrepublik mit ostdeutschen Wissenschaftlern verfährt ", in Die Zeit, 2. August 1996. 
Zusammenfassung: Am Beispiel des heutigen Geisteswissenschaftlichen Zentrums Moderner Orient, welches 1995 aus dem Forschungsschwerpunkt Moderner Orient hervorgegangen ist, das wiederum aus der Erbmasse der abgewickelten Akademie der Wissenschaften der DDR vier Jahre zuvor entstand, wird detailliert aufgezeigt, wie die Empfehlungen des Wissenschaftsrats der Bundesrepublik Deutschland vornehmlich in Bezug auf die empfohlene Weiterbeschäftigung der positiv evaluierten DDR-Wissenschaftler bis heute missachtet werden.

Stichworte: außeruniversitäre Geisteswissenschaften in der DDR, Abwicklung, deutsche Wiedervereinigung, Orient-, Asien- und Afrikawissenschaften in Deutschland

\section{The challenge of reconstructing the non-universitary humanities after the fall of the Berlin Wall. The case of the Research Centre for Modern Oriental Studies}

Summary: The Centre for Modern Oriental Studies (Geisteswissenschaftliches Zentrum Moderner Orient), which was created in 1996 from the Research Centre for Modern Oriental Studies (Forschungsschwerpunkt Moderner Orient), which in turn was created four years prior from the estate of the liquidated Academy of Sciences of the GDR, is exemplary for how recommendations of the Scientific Council of the Federal Republic of Germany that positively evaluated GDR scientists be retained have been ignored up to the present.

Keywords: non-university humanities in the GDR, completion, German unification, Oriental-, Asia- and Africa studies in Germany

Autor

Prof. Dr. Dr. Dr. Ulrich van der Heyden

Visiting Researcher

Department of Biblical and Ancient Studies of the University of South Africa (Unisa)

PO Box 392

Pretoria 0003

South Africa

h1107dpp@rz.hu-berlin.de 